\title{
Liver Metastases Arising from Well-Differentiated Pancreatic Endocrine Neoplasms Demonstrate Increased VEGF-C Expression
}

Donna E. Hansel, M.D., Ph.D., Ayman Rahman, John Hermans, MSc, M.D., Ronald R. de Krijger, M.D., Raheela Ashfaq, M.D., Charles J. Yeo, M.D., John L. Cameron, M.D., Anirban Maitra, M.D.

Departments of Pathology (DEH, AR, AM) and Surgery (CJY, JLC), Johns Hopkins University School of Medicine, Baltimore, Maryland; Departments of Pathology (DEH, RDK) and Radiology (JH), Erasmus Medical Center, Rotterdam, The Netherlands; and the Department of Pathology, University of Texas Southwestern Medical Center, Dallas, Texas (RA)

Pancreatic endocrine neoplasms (PENs) are uncommon, generally well-differentiated neoplasms that demonstrate prominent endocrine differentiation. Although the majority of PENs remain localized, malignant spread may occur via lymphatic or hematogenous routes. Angiogenic growth factors, including the vascular endothelial growth factor (VEGF) family, have been implicated in new vessel growth and hematogenous metastases, although this has not been studied in PENs. We therefore examined 19 primary well-differentiated PENs and 7 liver metastases to determine the expression of VEGF-A and its family member VEGF-C by immunolabeling analysis. VEGF-A immunoreactivity was evident only in scattered cells throughout all lesions. VEGF-C, however, demonstrated low-tomoderate expression in primary PENs by semiquantitative histoscore analysis (factor of labeling intensity by percentage of positive cells), with significantly increased expression in liver metastases (mean histoscore indices: primary PEN, 4.7 versus liver metastases, 9.5; Student's $\boldsymbol{t}$ test; $P=\mathbf{. 0 0 2 7 7 3 )}$ ). Microvascular density of primary PENs and liver metastases did not appear to linearly correlate with VEGF-C expression. Examination of the VEGF-Cspecific receptors VEGFR-2/KDR/Flk-1 and VEGFR3/Flt-4 demonstrated intense endothelial immunoreactivity for VEGFR-2, as well as VEGFR-2 and -3 expression on the majority of neoplastic cells, suggesting a possible role in autocrine/paracrine neo-

Copyright (c) 2003 by The United States and Canadian Academy of Pathology, Inc.

VOL. 16, NO. 7, P. 652, 2003 Printed in the U.S.A

Date of acceptance: May 1, 2003.

This work was partially funded through a generous grant from the family of Margaret Lee.

Address reprint requests to: Anirban Maitra, M.D., Department of Pathology, Johns Hopkins University School of Medicine, 720 Rutland Ave, Ross 632, Baltimore, MD 21205; e-mail: amaitral@jhmi.edu.

DOI: 10.1097/01.MP.0000077416.68489.50 plastic growth regulation. We postulate that the upregulation of VEGF-C may be involved in PEN progression and metastases, although not via a direct proangiogenic mechanism.

KEY WORDS: Angiogenesis, Blood vessel, Endothelium, Flk-1, Flt-4, Growth, KDR, Metastasis, Microvascular density, Pancreas, Pancreatic endocrine neoplasm, VEGF, VEGF-C.

Mod Pathol 2003;16(7):652-659

Pancreatic endocrine neoplasms (PENs) occur predominantly in adults and demonstrate endocrine differentiation with formation of electron-dense secretory granules (1). These granules frequently contain insulin, glucagon, somatostatin, or gastrin, which may produce an identifiable clinical syndrome upon release. Although the majority of PENs remain localized, hematogenous and lymphatic metastasis may occur. The WHO classification of PENs separates these lesions into welldifferentiated and poorly differentiated categories, with the distinguishing criterion as highly atypical cells (2); metastases are present in both categories.

In general, PENs demonstrate prominent vascularity, although little is known regarding angiogenic factors that promote new vessel formation in these neoplasms. Because angiogenesis influences both neoplastic development and risk of invasion, identification of angiogenic factors involved in PENs may provide further insight into the pathobiology of neoplastic growth and spread, as well as indicating a potential therapeutic target for these lesions $(3,4)$.

Recent work has identified a variety of new-vessel-promoting factors, including Ang-1, Ang-2, cyclophilin, PECAM-1, Tie-1, Tie-2, VE-Cadherin, vascular endothelial growth factor (VEGF) family members, and VEGF-specific receptors (VEGFRs) 
(4). Increases in VEGF/VEGF-A expression correlate with increased microvascular density in a large variety of neoplasms, including pancreatic ductal adenocarcinoma, breast carcinoma, and gastric carcinoma (5-9). Blockade of VEGF signaling via multiple anti-VEGF agents in a neuroblastoma xenograft model induces vascular regression and suggests a potent role of VEGF in neoplasmassociated angiogenesis (10).

VEGF belongs to the cysteine knot growth factor/ platelet-derived growth factor (PDGF) family, which includes VEGF (VEGF-A), VEGF-B, VEGF-C, VEGF-D, placenta-induced growth factor (PIGF), and the viral-specific VEGF-E (11). These proteins selectively interact with the receptors VEGFR-1 (Flt1), VEGFR-2 (KDR, Flk-1), and VEGFR-3 (Flt-4), which contain seven extracellular immunoglobulin homology domains, a transmembrane domain, and tyrosine kinase intracellular domain.

VEGF-C interacts specifically with VEGFR-2/ KDR/Flk-1 and VEGFR-3/Flt-4 (11). VEGFR-2 binds VEGF, VEGF-C, VEGF-D, and VEGF-E and can activate numerous downstream signaling pathways that function in promoting proliferation, survival, and cell migration (11). VEGFR-3 binds VEGF-C and VEGF-D and has been predominantly implicated in lymphangiogenesis, although evidence is accumulating that VEGFR-3 may also function in angiogenesis (12-15). In addition, several of the VEGF receptors have been implicated in proliferation of neoplastic cells via an autocrine loop $(16,17)$.

In this study, we examined 26 resection specimens, including 19 well-differentiated primary PENs and 7 liver metastases to determine the ex- pression of selected VEGF family members and their receptors in these lesions. VEGF-C expression was further characterized via histoscore analysis and comparison with microvascular density in all lesions.

\section{MATERIALS AND METHODS}

\section{Patients}

Permission for this study was received from the Johns Hopkins Joint Committee on Clinical Investigation. Paraffin-embedded material from a series of 19 pancreatectomies with well-differentiated PENs, treated at The Johns Hopkins Hospital (Baltimore, MD) between the years 1996 and 2001, were used for the study (Table 1). Three of these patients underwent surgery for a paired primary PEN and a liver metastasis, and in four cases, histologic material from liver metastases only was available. In addition, four paired lymph node metastases were also available for analysis. The slides were reviewed by two of the authors (DEH and AM) to confirm the diagnosis and to select appropriate paraffinembedded blocks for immunohistochemistry. Patient characteristics were as follows: median age, 55.0 years (range, 24-73 y) and 1:1.3 female-male ratio. Average primary PEN diameter was $6.7 \mathrm{~cm}$ (range, 1-18 cm). By light microscopy, 9/19 primary PENs demonstrated microscopic angioinvasion, and 2/19 demonstrated lymphatic invasion. Using the WHO classification, all neoplasms were well differentiated PENs; 3 were benign, 6 were of uncertain behavior, and 10 were low-grade malignant (2).

TABLE 1. Patient Data and PEN Description

\begin{tabular}{|c|c|c|c|c|c|c|c|c|}
\hline Case \# & Age (year) & Gender & Location & Size & Angioinv & Lymph inv & Mets & WHO \\
\hline 1 & 42 & M & Distal pancreas & $18 \mathrm{~cm}$ & Yes & No & No & U \\
\hline 2 & 24 & $\mathrm{~F}$ & Head of pancreas & $17.5 \mathrm{~cm}$ & Yes (portal v) & No & No & $\mathrm{U}$ \\
\hline 3 & 69 & M & Distal pancreas & $8 \mathrm{~cm}$ & No & No & $\mathrm{LN}$ & $\mathrm{L}$ \\
\hline 4 & 56 & $\mathrm{~F}$ & Distal pancreas & $1.9 \mathrm{~cm}$ & No & No & No & $\mathrm{B}$ \\
\hline 5 & 43 & $\mathrm{~F}$ & Head of pancreas & $2.5 \mathrm{~cm}$ & No & No & No & $\mathrm{U}$ \\
\hline 6 & 41 & M & Distal pancreas & $1.5 \mathrm{~cm}$ & No & No & No & B \\
\hline 7 & 69 & $\mathrm{~F}$ & Head of pancreas & $2.3 \mathrm{~cm}$ & Yes & No & No & $\mathrm{U}$ \\
\hline 8 & 73 & M & Distal pancreas & $1 \mathrm{~cm}$ & No & No & No & $\mathrm{B}$ \\
\hline 9 & 57 & $\mathrm{~F}$ & Distal pancreas & $7.5 \mathrm{~cm}$ & No & No & Liver, LN & $\mathrm{L}$ \\
\hline 10 & 58 & M & Distal pancreas & $13 \mathrm{~cm}$ & No & No & No & $\mathrm{U}$ \\
\hline 11 & 53 & $\mathrm{~F}$ & Distal pancreas & $5 \mathrm{~cm}$ & No & No & No & $\mathrm{U}$ \\
\hline 12 & 70 & M & Head of pancreas & $3.5 \mathrm{~cm}$ & Yes & No & $\mathrm{LN}$ & $\mathrm{L}$ \\
\hline 13 & 48 & M & Distal pancreas & $15 \mathrm{~cm}$ & No & No & $\mathrm{LN}$ & $\mathrm{L}$ \\
\hline 14 & 52 & $\mathrm{~F}$ & Uncinate process & $6 \mathrm{~cm}$ & Yes & Yes & Liver, LN & $\mathrm{L}$ \\
\hline 15 & 44 & M & Head of pancreas & $5.5 \mathrm{~cm}$ & Yes & No & $\mathrm{LN}$ & $\mathrm{L}$ \\
\hline 16 & 58 & M & Head of pancreas & $5 \mathrm{~cm}$ & No & No & $\mathrm{LN}$ & $\mathrm{L}$ \\
\hline 17 & 50 & $\mathrm{~F}$ & Distal pancreas & $4.5 \mathrm{~cm}$ & Yes & Yes & Liver, LN & $\mathrm{L}$ \\
\hline 18 & 38 & M & Distal pancreas & $7 \mathrm{~cm}$ & Yes & No & Liver, LN & $\mathrm{L}$ \\
\hline 19 & 54 & M & Head of pancreas & $2.5 \mathrm{~cm}$ & Yes & No & Liver & $\mathrm{L}$ \\
\hline 20 & 53 & M & Primary pancreas & & & & Liver* & \\
\hline 21 & 66 & $\mathrm{~F}$ & Primary pancreas & & & & Liver* & \\
\hline 22 & 70 & $\mathrm{~F}$ & Primary pancreas & & & & Liver* & \\
\hline 23 & 61 & M & Primary pancreas & & & & Liver* & \\
\hline
\end{tabular}

Patients 17-19 underwent surgery for both primary PEN and liver metastasis; Patients 20-23 underwent surgery for liver metastasis only; B = benign behavior, $\mathrm{U}=$ uncertain behavior, $\mathrm{L}=$ low-grade malignant. 


\section{Immunohistochemistry}

Three- to four-micrometer sections from paraffinembedded tissue were used for immunohistochemical labeling. Slides were deparaffinized in fresh xylenes and rehydrated through sequential graded ethanol steps. Antigen retrieval was performed by citrate buffer incubation (18 mM citric acid, $8.2 \mathrm{~mm}$ sodium citrate, $\mathrm{pH}$ 6.0) using a household vegetable steamer (Black and Decker) for 60 minutes. Slides were incubated for 5 minutes with $3 \%$ hydrogen peroxide, washed in TBS/T (20 mM Tris, $140 \mathrm{~mm} \mathrm{NaCl}$, $0.1 \%$ Tween-20, pH 7.6), and incubated in appropriate antibody dilutions for CD31 (1:80; DAKO, Carpinteria, CA), VEGF (1:100; Santa Cruz Biotechnology, Santa Cruz, CA), VEGF-C (1:75; Zymed, San Francisco, CA), VEGFR-3/Flt-4 (1:400; Chemicon, Temecula, CA), and VEGFR-2/KDR/Flk-1 (1:200; Santa Cruz Biotechnology) for 60 minutes at room temperature. The avidin-biotin-peroxidase complex method from DAKO (Glostrup, Denmark) was used, and slides were subsequently counterstained with hematoxylin.

\section{Counting Procedure}

CD31-positive vessels were used for assessment of vascular density. The area with the greatest CD31 density of vessels was identified, and counting was performed on $10 \times 1 \mathrm{~mm}^{2}$ fields using an Olympus BH-2 microscope (Olympus Optical, Japan), with the observer blinded to pathology data.

\section{Statistical Analysis}

Semiquantitative assessment of VEGF-C immunolabeling was performed by histoscore analysis (multiplication product of percentage of positive cells by staining intensity) (18). Percentage of positive cells was evaluated as follows: $0,<5 \%$ labeled cells; $1,5-25 \%$ labeled cells; 2, 26-50\% labeled cells; $3,51-75 \%$ labeled cells; and $4,>75 \%$ labeled cells. The intensity of labeling was evaluated, with a range from 0 to 3 . By definition, the maximum positive score possible is 12. Values for CD31 counts are listed as average \pm standard deviation. $P$ values for paired metastases were calculated using a twotailed Student's $t$ test for samples of unequal variance.

\section{RESULTS}

\section{VEGF-A Expression Is Limited in Pancreatic Endocrine Neoplasms}

Because VEGF-A has been implicated as a prominent factor in neoplasm-associated angiogenesis, we first examined the expression of VEGF-A in both primary PENs and liver metastases. Interestingly, VEGF-A expression was limited to scattered cells within the primary neoplastic mass and metastatic lesions (data not shown). No correlation between VEGF-A expression and MVD was apparent within these specimens.

\section{VEGF-C Demonstrates Variable Expression in Primary PENs But Is Highly Expressed in Liver Metastases}

Because VEGF-A expression appeared not to be highly expressed in PENs, we next examined VEGF-C protein expression in 19 primary welldifferentiated PENs and 7 liver metastases. Within primary PENs, VEGF-C immunolabeling was variable (Fig. 1A), with a mean histoscore labeling index of 4.7. The majority of these lesions demonstrated an intensity of 1, with $26-100 \%$ of cells labeled (Fig. 1A); occasional primary lesions, however, obtained a higher histoscore, with a labeling intensity of $2-3$ and $>50 \%$ of cells demonstrating immunoreactivity (Fig. 1B). Subclassification of primary PENs into benign behavior $(n=3)$, uncertain behavior $(n=6)$, and low-grade malignant behavior ( $n=10$ ) using published WHO criteria (2) yielded respective histoscore indices of 1.3, 5.2, and 5.5. Application of secondary antibody alone yielded no immunostaining.

In contrast to the lower levels of VEGF-C immunolabeling in primary PENs, liver metastases demonstrated greatly increased intensity of immunolabeling, with virtually all cells of the metastatic lesion demonstrating immunoreactivity (Fig. 2, A versus $\mathrm{B})$. The mean histoscore index for liver metastases was 9.5, with the majority of lesions demonstrating an intensity of 2 or 3 in $>75 \%$ of cells. A Student's $t$ test comparing primary PENs with liver metastases yielded a $P$ value of .002773 .

\section{VEGF-C Levels Do Not Appear to Directly} Correlate with Microvascular Density in Primary Lesions or Liver Metastases

Because VEGF-C previously has been described as an angiogenic factor, we next assessed the relationship between VEGF-C expression and microvascular density within both primary PENs and liver metastases. Within both primary and metastatic lesions, microvascular density varied from as low as 12 blood vessels $/ \mathrm{mm}^{2}$ to as high as 180 blood vessels $/ \mathrm{mm}^{2}$ (Fig. 3,A-C). Although the variation within each lesion was minimal, with small standard errors of the mean (error bars, Fig. 3C), no direct correlation was apparent for either MVD or MVD versus VEGF-C between primary lesions and metastases. Analysis of individual lesions, however, did reveal that lesions with extremely low VEGF-C histoscore indices correlated with lower MVD and vice versa. For example, the primary PEN with 12 

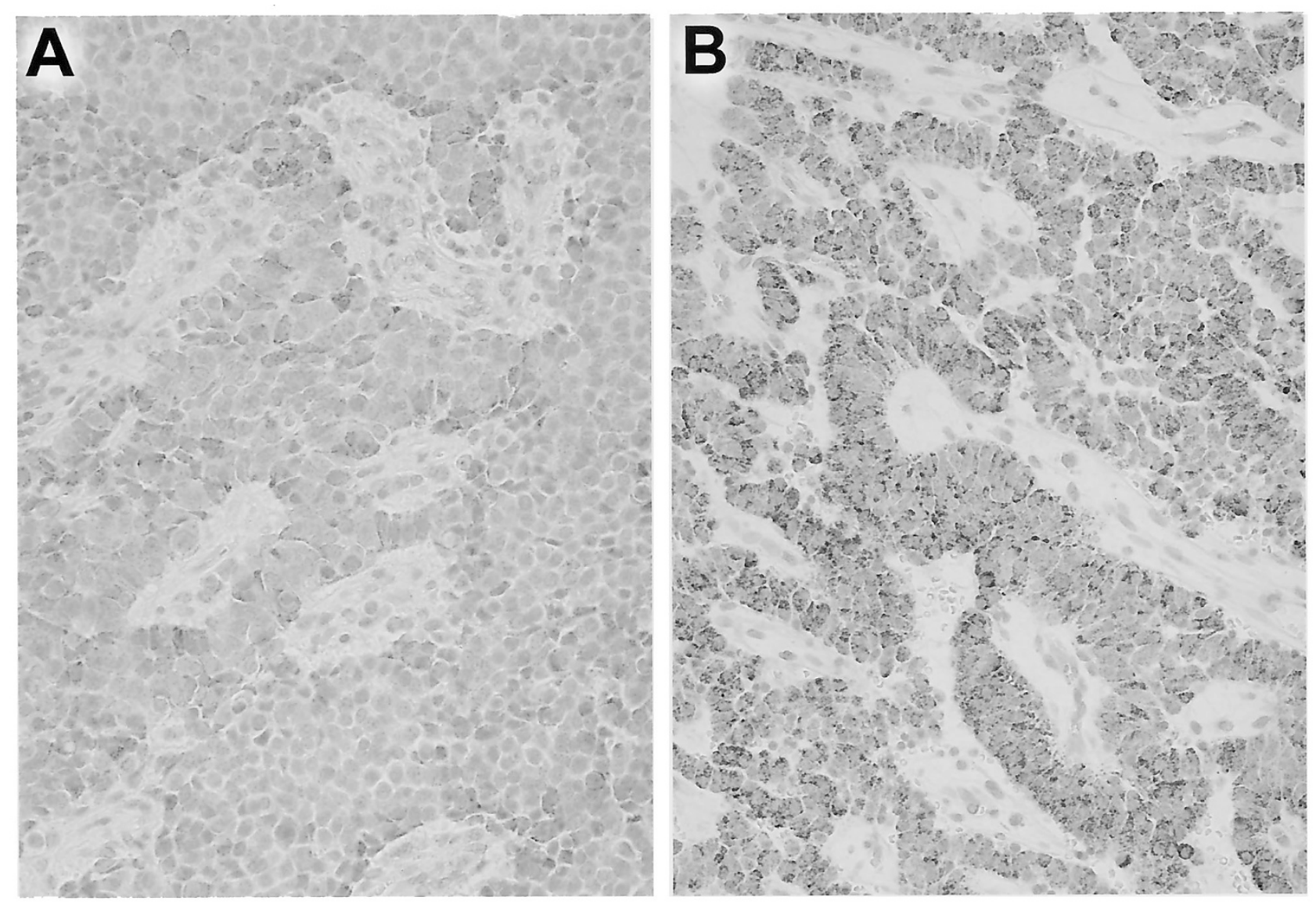

FIGURE 1. VEGF-C expression in primary PENs. A, lesion with low VEGF-C expression in neoplastic cells and with B, high VEGF-C expression in neoplastic cells. Magnification, $160 \times$.

blood vessels $/ \mathrm{mm}^{2}$ demonstrated a histoscore index of 1 , whereas the liver metastasis with 180 blood vessels $/ \mathrm{mm}^{2}$ demonstrated a histoscore index of 8 .

\section{Endothelial cells Express High Levels of VEGFR-} 2, But Only Low Levels of VEGFR-3

Although VEGF-C expression does not demonstrate a significant linear correlation with MVD, VEGF-C may still function to promote vascular growth within PENs by binding to and activating the receptors VEGFR-2/KDR/Flk-1 and VEGFR-3/ Flt- 4 on endothelial cells. We therefore examined a subset of PENs (8 primary PENs and 3 liver metastases) for VEGFR-2 and -3 expression on endothelial cells. Immunolabeling for VEGFR-3 demonstrated only scattered weak positivity in endothelial cells, regardless of PEN location or level of VEGF-C expression (Fig. 4A). In comparison, high levels of immunoreactivity for VEGFR-2 were seen in endothelial cells of all lesions examined (Fig. 4B), suggesting that VEGFR-2 mediates the effects of VEGF-C on angiogenesis in pancreatic endocrine neoplasms.

\section{Pancreatic Endocrine Neoplasm Cells Demonstrate VEGFR-2 and -3 Expression}

Although VEGFR-2 appears to be highly expressed on endothelial cells, both VEGFR-2 and -3 were also variably expressed by neoplastic cells. These receptors have been previously suggested to mediate an autocrine function of the VEGF family members on neoplastic growth. Comparison of VEGF-C expression with VEGFR-2 and -3 revealed a potential correlation between receptor and ligand expression in primary PENs and liver metastases, although further studies are necessary with additional lesions to determine whether this correlation is significant. These results, however, indicate that VEGFR-2 and -3 may be potentially involved in regulating either PEN growth or survival.

\section{DISCUSSION}

VEGF family members belong to the cysteine knot growth factor/platelet-derived growth factor (PDGF) family and promote both angiogenesis and lymphangiogenesis, depending on which isoform of receptor is expressed on the target endothelium (11). VEGF-A has been shown to be expressed in 

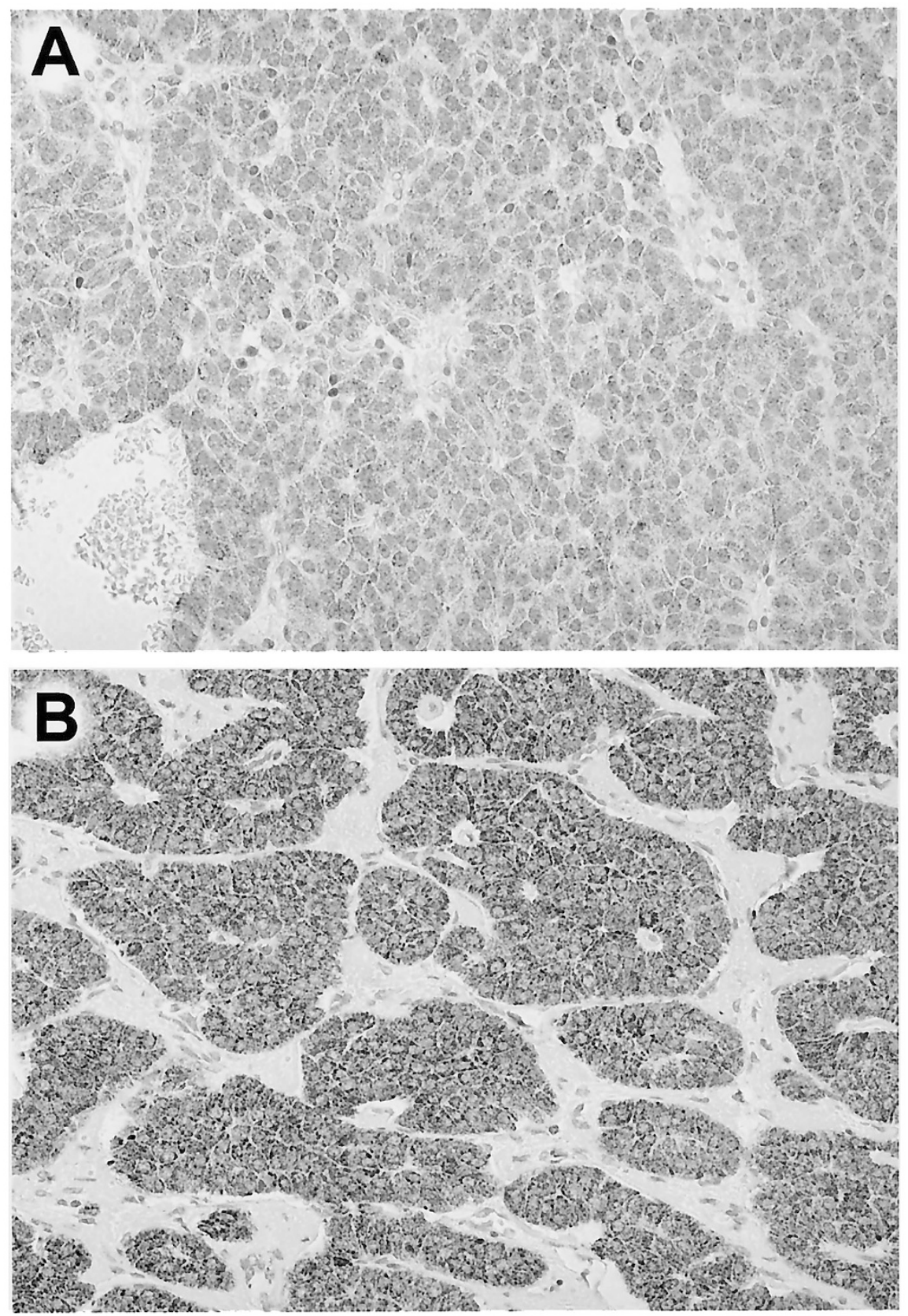

FIGURE 2. VEGF-C expression in a primary PEN and paired liver metastasis. A, primary pancreatic endocrine neoplasm with $\mathbf{B}$, paired liver metastasis showing increased VEGF-C expression in the liver metastasis. 160×.

normal islets of the pancreas and to mediate angiogenesis in several neoplasms $(7,9,19)$. In our study, however, VEGF-A expression appears to be limited to scattered cells within PENs and is not correlated with MVD.

VEGF-C, however, appears to be variably expressed in primary PENs. Of note, separation into WHO subclassifications of well-differentiated PENs (2) reveals a low VEGF-C histoscore index for PENs of benign behavior and a relatively higher histoscore index for PENs of either uncertain or low-grade malignant behavior. These findings suggest that VEGF-C expression may be in- volved in the progression of primary welldifferentiated PENs, and VEGF-C expression may be used as an adjunct measure in the subclassification of these lesions.

The apparent increase in VEGF-C expression in liver metastases supports the possible role of VEGF-C in neoplastic PEN progression. An interesting correlate to this finding was the identification of the relative down-regulation of VEGF-C in lymph node metastases in comparison with primary PEN lesions, although the number of lymph node metastases was too small for statistical significance ( $n$ $=4$; data not shown). These findings also suggest 

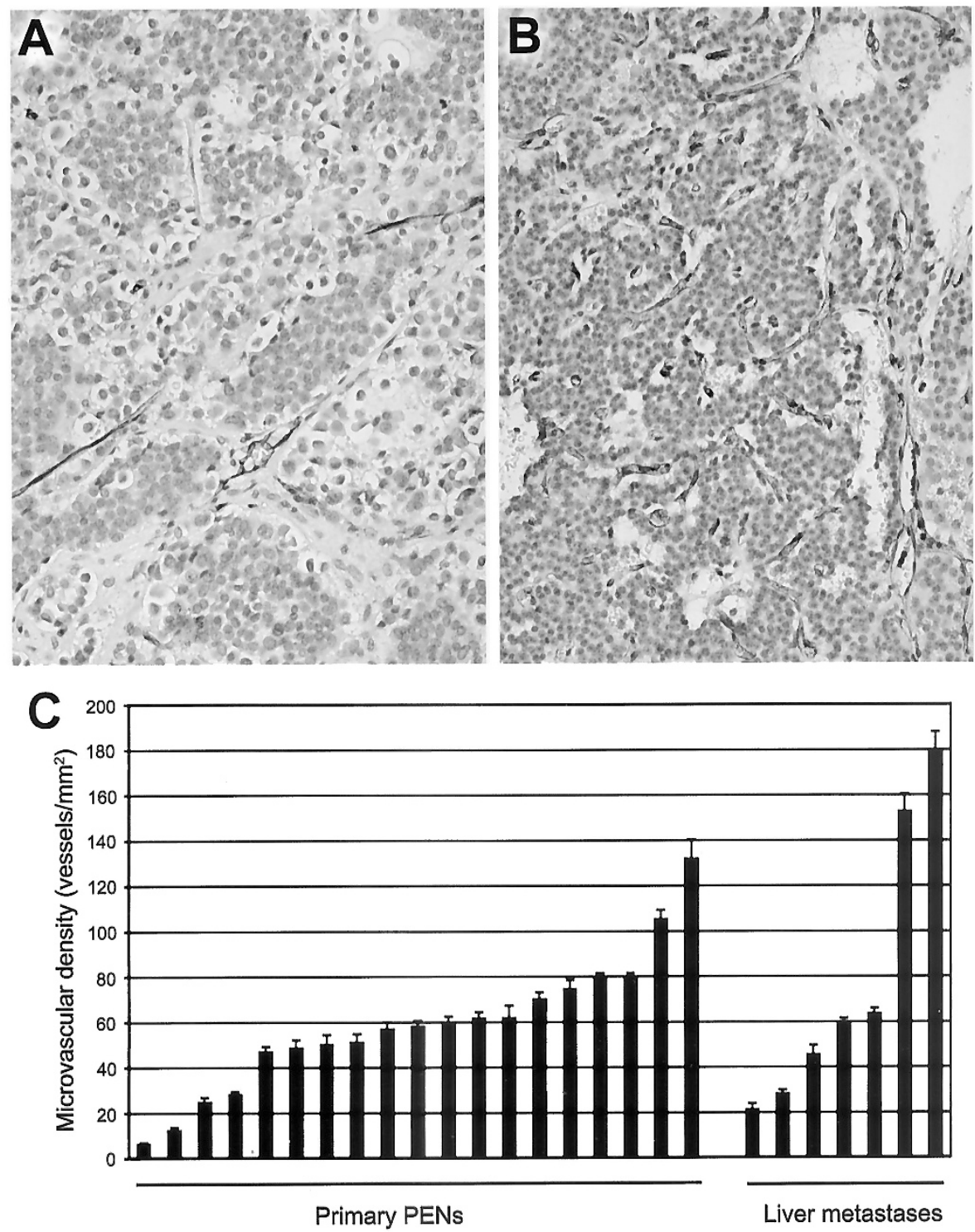

FIGURE 3. CD31 immunolabeling of primary pancreatic endocrine neoplasms. A, lesion with low vascularity and B, a lesion with high vascularity. $160 \times$. C, microvascular density of primary PENs and liver metastases.

that VEGF-C expression may also be directly influenced by locally-produced factors in the environment surrounding the metastatic lesion. Further studies using larger numbers of metastatic PENs may be useful in addressing this issue.

In our study, we have identified VEGFR-2 as the major VEGF-C receptor expressed on endothelial cells in PENs. VEGF-C binds to VEGFR-2 and -3, and the specificity of VEGF-C for these receptors appears to be mediated by posttranslational proteolytic processing of VEGF-C (20). Increased VEGF-C expression has been primarily studied in neoplastic processes as a mediator of angiogenesis, and the VEGFR-2 receptor expressed on endothelial cells has been indicated as the major receptor required for new vessel growth (11). VEGFR-2 has been reported to induce angiogenesis in a number of neoplasms, including glioblastoma, endometrial cancer, and breast carcinoma, as well as to promote revascularization in transplanted rat islets (21-24). Although no statistically significant association between MVD and VEGF-C expression was evident in our study of PENs, VEGF-C may still influence new vessel growth in a non-dose-dependent manner or by levels of proteolytic processing, which was not addressed in this study.

An additional finding in our study was the identification of VEGFR-2 and -3 expression by neoplastic cells. Activation of VEGF receptors by ligand induces dimerization and tyrosine phosphorylation with subsequent activation of protein kinase C, PI3kinase, and Ras pathways (11). Although these pathways have been studied in relationship to endothelial cell proliferation, survival, and migration, they may also serve to promote a similar function in neoplastic cells. In support of this hypothesis, VEGF 

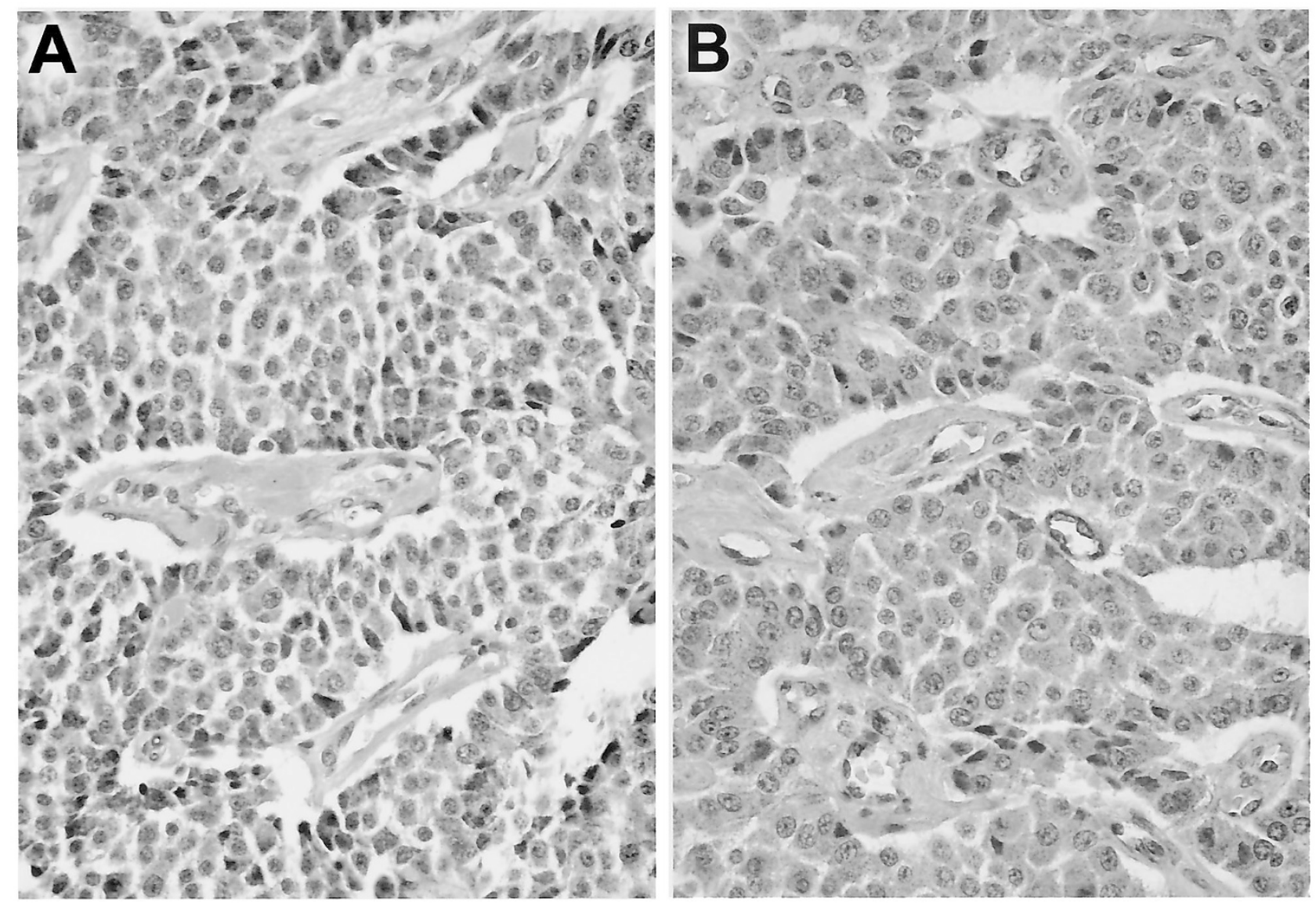

FIGURE 4. Comparison of VEGFR-3 and -2 immunolabeling in a primary pancreatic endocrine neoplasm. A, low expression of VEGFR-3 in endothelial cells. B, VEGFR-2 demonstrates high expression in neoplasm-associated endothelial cells. Serial sections from the same PEN were used, with comparison of matched fields. Note expression of both receptor types by neoplastic cells.

receptors have been reported to be expressed by a number of other neoplastic cell types, including choriocarcinoma and melanoma cells, and inhibition of VEGFRs by neutralizing antibodies can inhibit proliferation of neoplastic cells in vitro (17, 25-27). VEGFR-2 has also been shown to be expressed by RINm5F cultured pancreatic endocrine cells, fetal rat islets, and in cells lining the pancreatic ducts, which have been proposed to represent precursor cells of the endocrine pancreas (28).

The increased expression of VEGF-C in liver metastases of PENs relative to primary PENs, with concomitant expression of its receptors on both endothelium and neoplastic cells, suggests that VEGF-C may function not only to promote a certain level of angiogenesis but also to promote neoplastic growth. In addition, the apparent upregulation of VEGF-C expression from benign lesions to those of uncertain and low-grade malignant potential suggests that VEGF-C may be involved in PEN progression. Further studies are necessary to determine the potential role of VEGFR-2 and -3 in cancer growth, as well as to identify the signaling pathways that may be involved in inducing and mediating the expression of VEGF-C within these lesions.
Acknowledgments: We would like to thank Frieda van der Ham in the Department of Pathology, JNI, Erasmus Medical Center, Rotterdam, the Netherlands, for her outstanding technical support.

\section{REFERENCES}

1. Gumbs AA, Moore PS, Falconi M, et al. Review of the clinical, histological, and molecular aspects of pancreatic endocrine neoplasms. J Surg Oncol 2002;81(1):45-53; discussion 54.

2. Solcia E, Kloppel G, Sobin LH, editors. Histological typing of endocrine tumours. New York: Springer-Verlag; 2001.

3. Kubota Y, Kleinman HK, Martin GR, Lawley TJ. Role of laminin and basement membrane in the morphological differentiation of human endothelial cells into capillary-like structures. J Cell Biol 1988;107(4):1589-98.

4. Shih SC, Robinson GS, Perruzzi CA, et al. Molecular profiling of angiogenesis markers. Am J Pathol 2002;161(1):35-41.

5. Niedergethmann M, Hildenbrand R, Wostbrock B, et al. High expression of vascular endothelial growth factor predicts early recurrence and poor prognosis after curative resection for ductal adenocarcinoma of the pancreas. Pancreas 2002; 25(2):122-9.

6. Seo Y, Baba H, Fukuda T, et al. High expression of vascular endothelial growth factor is associated with liver metastasis and a poor prognosis for patients with ductal pancreatic adenocarcinoma. Cancer 2000;88(10):2239-45. 
7. Lantzsch T, Hefler L, Krause U, et al. The correlation between immunohistochemically-detected markers of angiogenesis and serum vascular endothelial growth factor in patients with breast cancer. Anticancer Res 2002;22(3):1925-8.

8. Toi M, Inada K, Suzuki H, Tominaga T. Tumor angiogenesis in breast cancer: its importance as a prognostic indicator and the association with vascular endothelial growth factor expression. Breast Cancer Res Treat 1995;36(2):193-204.

9. Song ZJ, Gong P, Wu YE. Relationship between the expression of iNOS, VEGF, tumor angiogenesis and gastric cancer. World J Gastroenterol 2002;8(4):591-5.

10. Kim ES, Serur A, Huang J, et al. Potent VEGF blockade causes regression of coopted vessels in a model of neuroblastoma. Proc Natl Acad Sci U S A 2002.

11. Karkkainen MJ, Petrova TV. Vascular endothelial growth factor receptors in the regulation of angiogenesis and lymphangiogenesis. Oncogene 2000;19(49):5598-605.

12. Kaipainen A, Korhonen J, Mustonen T, et al. Expression of the fms-like tyrosine kinase 4 gene becomes restricted to lymphatic endothelium during development. Proc Natl Acad Sci U S A 1995;92(8):3566-70.

13. Stacker SA, Achen MG, Jussila L, et al. Metastasis. Lymphangiogenesis and cancer metastasis. Nat Rev Cancer 2002; 2(8):573-83.

14. Cao Y, Linden P, Farnebo J, et al. Vascular endothelial growth factor $\mathrm{C}$ induces angiogenesis in vivo. Proc Natl Acad Sci U S A 1998;95(24):14389-94.

15. Witmer AN, van Blijswijk BC, Dai J, et al. VEGFR-3 in adult angiogenesis. J Pathol 2001;195(4):490-7.

16. Dias S, Choy M, Alitalo K, Rafii S. Vascular endothelial growth factor (VEGF)-C signaling through FLT-4 (VEGFR-3) mediates leukemic cell proliferation, survival, and resistance to chemotherapy. Blood 2002;99(6):2179-84.

17. Masood R, Cai J, Zheng T, et al. Vascular endothelial growth factor (VEGF) is an autocrine growth factor for VEGF receptor-positive human tumors. Blood 2001;98(6):1904-13.

18. Scheres HM, De Goeij AF, Rousch MJ, et al. Quantification of oestrogen receptors in breast cancer: radiochemical assay on cytosols and cryostat sections compared with semiquantitative immunocytochemical analysis. J Clin Pathol 1988;41(6): 623-32.

19. Christofori G, Naik P, Hanahan D. Vascular endothelial growth factor and its receptors, flt- 1 and flk-1, are expressed in normal pancreatic islets and throughout islet cell tumorigenesis. Mol Endocrinol 1995;9(12):1760-70.

20. Joukov V, Sorsa T, Kumar V, et al. Proteolytic processing regulates receptor specificity and activity of VEGF-C. EMBO J 1997;16(13):3898-911.

21. Millauer B, Shawver LK, Plate KH, et al. Glioblastoma growth inhibited in vivo by a dominant-negative Flk-1 mutant. Nature 1994;367(6463):576-9.

22. Breier G, Blum S, Peli J, et al. Transforming growth factorbeta and Ras regulate the VEGF/VEGF-receptor system during tumor angiogenesis. Int J Cancer 2002;97(2):142-8.

23. Sivridis E. Angiogenesis and endometrial cancer. Anticancer Res 2001;21(6B):4383-8.

24. Vasir B, Jonas JC, Steil GM, et al. Gene expression of VEGF and its receptors Flk-1/KDR and Flt-1 in cultured and transplanted rat islets. Transplantation 2001;71(7):924-35.

25. Charnock-Jones DS, Sharkey AM, Boocock CA, et al. Vascular endothelial growth factor receptor localization and activation in human trophoblast and choriocarcinoma cells. Biol Reprod 1994;51(3):524-30.

26. Itakura J, Ishiwata $\mathrm{T}$, Shen $\mathrm{B}$, et al. Concomitant overexpression of vascular endothelial growth factor and its receptors in pancreatic cancer. Int J Cancer 2000;85(1):2734.

27. Liu B, Earl HM, Baban D, et al. Melanoma cell lines express VEGF receptor KDR and respond to exogenously added VEGF. Biochem Biophys Res Commun 1995;217(3): 721-7.

28. Oberg C, Waltenberger J, Claesson-Welsh L, Welsh M. Expression of protein tyrosine kinases in islet cells: possible role of the Flk-1 receptor for beta-cell maturation from duct cells. Growth Factors 1994;10(2):115-26. 